\title{
MEASUREMENT OF RIPPLE IN THE ACCUMULATOR BEND BUS SUPPLY
}

\author{
M. Church
}

31 January 1992

\section{"I. Introduction}

E760 uses the longitudinal Schottky spectrum of the circulating beam in the Accumulator to measure the momentum spectrum of the beam with the assumption that $\Delta p / p=\frac{1}{\eta} * \Delta f / f$. However, the presence of low frequency ripple in the bus current could modulate this spectrum so that the above relation is not true. Therefore an attempt was made to measure this ripple to see if it had a significant affect on the frequency spectrum.

\section{Calculation}

The longitudinal Schottky current at the pickup $P$ is given by

$$
I^{P}(t)=q \sum_{j=1}^{N} \sum_{m=-\infty}^{+\infty} \omega_{j} \cdot \delta\left(\theta_{j}(t)-\theta_{P}-2 m \pi\right)
$$

where

$$
\omega_{j}=\omega_{0 j}+A \omega_{R} \cos \left(\omega_{R} t\right)
$$

and

$$
\theta_{j}(t)=\omega_{0 j} t+A \sin \left(\omega_{R} t\right)+\psi_{j}^{0} .
$$

The sum $j$ is over the $N$ beam particles, $A$ is the amplitude of the ripple (in radians), $\omega_{R}$ is the ripple frequency, and $\theta_{P}$ is the azimuthal location of the pickup. Using the Fourier series representation of the periodic $\delta$ function, this becomes

$$
I^{P}(t)=q \sum_{j=1}^{N} \frac{\omega_{j}}{2 \pi} \sum_{m=-\infty}^{\infty} e^{-i m\left(\omega_{0 j} t-\theta_{j}^{0}\right)} \cdot e^{-i m A \sin \left(\omega_{R} t\right)}
$$

where

$$
\theta_{j}^{0}=\theta_{P}-\psi_{j}^{0}
$$

Using the bessel function relation

$$
e^{-i x \sin y}=\sum_{k=-\infty}^{\infty} J_{k}(x) e^{-i k y}
$$


yields

$$
I_{P}(t)=\frac{q}{2 \pi} \sum_{j=1}^{\infty} \omega_{0 j} e^{-i m\left(\omega_{0 j} t-\theta_{j}^{0}\right)} \sum_{k=-\infty}^{\infty} J_{k}(m A) e^{-i k \omega_{R} t}
$$

where I have used $\omega_{0 j} \simeq \omega_{j}$.

The autocorrelation function is

$$
C(\tau)=\lim _{T \rightarrow \infty} \frac{1}{2 T} \int_{-T}^{+T}<I^{P}(t) I^{P}(t+\tau)>d t
$$

where the average is over $\theta$. After averaging, analyzing $\delta$ functions, and taking the limit, this becomes

$$
C(\tau)=\frac{q^{2}}{(2 \pi)^{2}} \sum_{j k m} \omega_{0 j}^{2} J_{k}^{2}(m A) e^{i m \omega_{0} \tau} e^{i k \omega_{R} \tau} .
$$

The power spectrum is then

$$
P(\Omega)=\int_{-\infty}^{+\infty} d \tau \frac{q^{2}}{(2 \pi)^{2}} \sum_{j k m} \omega_{0 j}^{2} J_{k}^{2}(m A) e^{i\left(m \omega_{0 j}+k \omega_{R}-\Omega\right) t} .
$$

Let

$$
\sum_{j} \longrightarrow N \int d \omega_{0} \Psi\left(\omega_{0}\right) \text { where } \int_{-\infty}^{+\infty} d \omega_{0} \Psi\left(\omega_{0}\right)=1
$$

so that

$$
P(\Omega)=\sum_{k m} \frac{N q^{2}}{2 \pi m^{3}} J_{k}^{2}(m A)\left(\Omega-k \omega_{R}\right)^{2} \Psi\left(\frac{\Omega-k \omega_{R}}{m}\right) .
$$

For the mth harmonic this is

$$
\begin{aligned}
& P_{m}(\Omega)=\frac{N q^{2}}{2 \pi m^{3}} J_{0}^{2}(m A) \Omega^{2} \Psi\left(\frac{\Omega}{m}\right)+ \\
& \frac{N q^{2}}{2 \pi m^{3}} \sum_{k=1}^{\infty} J_{k}^{2}(m A)\left[\left(\Omega-k \omega_{R}\right)^{2} \Psi\left(\frac{\Omega-k \omega_{R}}{m}\right)+\left(\Omega+k \omega_{R}\right)^{2} \Psi\left(\frac{\Omega+k \omega_{R}}{m}\right)\right] .
\end{aligned}
$$

$A$ is given by

$$
A=\frac{\omega_{0}}{\omega_{R}} \frac{\Delta B}{B} \frac{1}{\gamma_{T}^{2}}
$$

where $B$ is the magnetic field and $\Delta B$ is the magnitude of the magnetic field ripple.

\section{Measurement}

In order to measure the ripple in the Accumulator bend field a wire coil was placed inside the dipole magnet which also contains the NMR probe. The frequency power spectrum was then measured at several different magnetic field values. A typical frequency spectrum is shown in Fig. 1. The voltage generated across the coil is

$$
V=-\frac{d B}{d t} B \pi R^{2} N
$$

where $N$ is the number of turns (100) and $R$ is the radius $(.05 \mathrm{~m})$. If we take

$$
B=B_{0}+\Delta B \cos \left(\omega_{R} t\right)
$$


then the RMS power into $50 \Omega$ (in watts) is

$$
P_{r m s}=.1396\left(\Delta B \omega_{R} R^{2} N\right)^{2},
$$

and the magnitude of the magnetic field ripple can be extracted from plots such as Fig. 1. The signal was carried from the tunnel to the AP30 service building on a twinaxial cable and the measurement was made differentially on an HP Dynamic Signal Analyzer.

Table 1 summarizes the data taken. The noise ( 0 field) measurements are well below the signals measured with the bend bus on, although there is no clear relationship between magnetic field strength and ripple size. The source of this ripple is not clear and it is not necessarily coming from the magnetic field - since the coil itself was not well shielded. Therefore, these measurements should be taken as an upper limit on ripple size. In addition, a stainless steel beampipe will partially filter out low frequency magnetic oscillations, therefore the measurements presented here must be considered upper limits for this reason also.

The magnitude of $\Delta B / B$ for the different measured cases is tabulated in Table 2. The calculation uses eq. 17. Table 3 shows the calculated affect of ripple on the width of the beam using eq. 13. The 3 magnetic field cases are $1.67,1.12$, and 0.77 Tesla. For each magnetic field case 4 different unperturbed beam widths (sigma) are considered $-140,160,180$, and $200 \mathrm{~Hz} @$ harmonic 127 which weré typical widths during the double scans. $\sigma$ is the unperturbed beam width, $\sigma_{1}$ is the ripple-perturbed sigma, and $\sigma_{2}$ is the ripple-perturbed $2 \mathrm{x}$ sigma. The last column is the percent change in the beam sigma due to the ripple. The worst case is $.14 \%$ which is entirely negligible for E760's purposes. 
Bfield (gauss) -- A:IB (amps)

freq. $(\mathrm{Hz}) 16752-1171 \quad 11161-7457718-513 \quad 0-0$

$\begin{array}{rrrrr}60 & -47.3 & -47.6 & -51.8 & -100.9 \\ 120 & -46.0 & -41.8 & -47.9 & -111.8 \\ 180 & -38.6 & -52.9 & -48.4 & -101.9 \\ 240 & -53.6 & -50.1 & -55.7 & -113.8 \\ 300 & -53.2 & -53.4 & -56.0 & -92.3 \\ 360 & -43.9 & -39.4 & -36.6 & -94.4 \\ 420 & -60.8 & -58.8 & -56.7 & -94.8 \\ 480 & -59.0 & -60.6 & -61.9 & -105.8 \\ 540 & -52.8 & -54.1 & -59.7 & -104.8 \\ 600 & -64.1 & -61.3 & -62.3 & -103.9 \\ 660 & -59.7 & -64.0 & -63.1 & -92.6 \\ 720 & -36.1 & -33.0 & -32.5 & -94.7\end{array}$

TABLE 1: measured power (dBm) for different frequency and Bfield values 
From FNWSO7: USR SROOTI: [CNTURCH.ACNET] JRIPPLEZ-HOK

$B=1.67$ tes $1 a$

$\mathrm{F}=60 . \mathrm{hz}$ $-F=120 . \mathrm{hz}$ $=180 . h z$ $F=240 . h z$ $F=300$. hz $\mathrm{F}=360$. hz $F=420$. hz $F=480$. hz $\mathrm{F}=540$. hz $\mathrm{F}=600$. hz $\mathrm{F}=660$, hz $F=720$. hz
$D B / B=0.0000023$ $D B / B=0.0000013$ $\mathrm{DB} / \mathrm{B}=0.0000021$ $\mathrm{DB} / \mathrm{B}=0.0000003$ $D B / B=0.0000002$ $D B / B=0.0000006$ $D B / B=0.0000001$ $D B / B=0.0000001$ $D B / B=0.0000001$ $D B / B=0.0000000$ $D B / B=0.0000001$ $D B / B=0.0000007$
Vpeak $=1.147 \mathrm{mv}$ Vpeak $=1.333 \mathrm{mv}$ Vpeak $=3.124 \mathrm{mv}$ Vpeak $=0.556 \mathrm{mv}$ Vpeak $=0.582 \mathrm{mv}$ Ypeak $=1.697 \mathrm{mv}$ $V_{\text {peak }}=0.243 \mathrm{mv}$ $V_{\text {peak }}=0.298 \mathrm{mv}$ Vpeak $=0.609 \mathrm{mv}$ $V_{\text {peak }}=0.166 \mathrm{mv}$ Vpeak $=0.275 \mathrm{mv}$ Vpeak $=4.166 \mathrm{mv}$
Prms $=0.186 \mathrm{E}-04 \mathrm{mw}$ Prms $=0.251 \mathrm{E}-04 \mathrm{mw}$ Prms $=0.138 E-03 \mathrm{mw}$ Prms $=0.437 \mathrm{E}-05 \mathrm{mw}$ Prms $=0.479 \mathrm{E}-05 \mathrm{mw}$ Prms $=0.407 \mathrm{E}-04 \mathrm{mw}$ Prms $=0.832 E-06 \mathrm{mw}$ Prms $=0.126 \mathrm{E}-05 \mathrm{mw}$ Prms $=0.525 E-05 \mathrm{mw}$ Prms $=0.389 \mathrm{E}-06 \mathrm{mw}$ Prms $=0.107 E-05 . \mathrm{mw}$ Prms $=0.245 E-03 \mathrm{mw}$

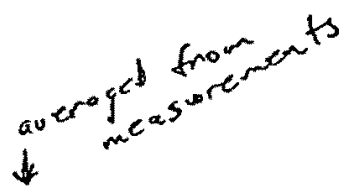

$\mathrm{dBm}=-47.3$

$\mathrm{dB} m=-46.0$

$\mathrm{dBm}=-38.6$

$\mathrm{dBm}=-53.6$

$\mathrm{dBm}=-53.2$

$\mathrm{dB} m=-43.9$

$\mathrm{dBm}=-60.8$

$\mathrm{dBm}=-59.0$

$\mathrm{dBm}=-52.8$

$\mathrm{dBm}=-64.1$

$\mathrm{dBm}=-59.7$

$\mathrm{dBm}=-36.1$

$B=1.12$ tes $1 \mathrm{a}$

$\mathrm{F}=60 . \mathrm{hz}$

$F=120 . h z$

$F=180 . h z$

$F=240 . h z$

$F=300$. hz

$F=360$. hz

$F=420$, hz

$F=480 . \mathrm{hz}$

$F=540 . h z$

$F=600 . \mathrm{hz}$

$F=660$. hz $\mathrm{F}=720 \mathrm{hz}$
$D B / B=0.0000033$

$D B / B=0.0000033$

$D B / B=0.0000006$

$D B / B=0.0000006$

$D B / B=0.0000003$

$D B / B=0.0000014$

$D B / B=0.0000001$

$D B / B=0.0000001$

$D B / B=0.0000002$

$D B / B=0.0000001$

$D B / B=0.0000000$

$D B / B=0.0000015$
Vpeak $=1.109 \mathrm{mv}$ Vpeak $=2.161 \mathrm{mv}$ $V_{\text {peak }}=0.602 \mathrm{mv}$ $V_{\text {peak }}=0.831 \mathrm{mv}$ Vpeak $=0.569 \mathrm{mv}$ Vpeak $=2.849 \mathrm{mv}$ $V_{\text {peak }}=0.305 \mathrm{mv}$ Vpeak $=0.248 \mathrm{mv}$ Veak $=0.524 \mathrm{mv}$ vpeak $=0.229 \mathrm{mv}$ Vpeak $=0.168 \mathrm{mv}$ Vpeak $=5.953 \mathrm{mv}$
Prms $=0.174 \mathrm{E}-04 \mathrm{mw}$ Prms $=0.661 \mathrm{E}-04 \mathrm{mw}$ Prms $=0.513 E-05 \mathrm{mw}$ Prms $=0.977 \mathrm{E}-05 \mathrm{mw}$ Prms $=0.457 \mathrm{E}-05 \mathrm{mw}$ Prms $=0.115 E-03 \mathrm{mw}$ Prms $=0.132 \mathrm{E}-05 \mathrm{mw}$ Prms $=0.871 \mathrm{E}-06 \mathrm{mw}$ Prms $=0.389 E-05 \mathrm{mw}$ Prms $=0.741 \mathrm{E}-06 \mathrm{mw}$ Prms $=0.398 \mathrm{E}-06 \mathrm{mw}$ Prms $=0.501 E-03 \mathrm{~mm}$
$\mathrm{dBm}=-47.6$ $\mathrm{dB} m=-41.8$ $\mathrm{dBm}=-52.9$ $\mathrm{dBm}=-50.1$ $\mathrm{dBm}=-53.4$ $\mathrm{dBm}=-39.4$ $\mathrm{dBm}=-58.8$ $\mathrm{dBm}=-60.6$ $\mathrm{dBm}=-54.1$ $\mathrm{dB} m=-61.3$ $d B m=-64.0$ $\mathrm{dBm}=-33.0$

$b=0.77$ tes $1 a$

$\mathrm{F}=60 . \mathrm{hz}$ $\mathrm{F}=120 . \mathrm{hz}$ $\mathrm{F}=180 . \mathrm{hz}$ $F=240$. hz $\mathrm{F}=300 . \mathrm{hz}$ $\mathrm{F}=360 . \mathrm{hz}$ $F=420$. hz $F=480$. hz $F=540 . h z$ $F=600 . h z$ $F=660$. hz $F=720$. hz
$D B / B=0.0000030$ $D B / B=0.0000023$ $D B / B=0.0000015$ $D B / B=0.0000005$ $D B / B=0.0000004$ $D B / B=0.0000029$ $D B / B=0.0000002$ $D B / B=0.0000001$ $D B / B=0.0000001$ $D B / B=0.0000001$ $D B / B=0.0000001$ $D B / B=0.0000023$
Vpeak $=0.684 \mathrm{mv}$ veak $=1.071 \mathrm{mv}$ veak $=1.011 \mathrm{mv}$ $V_{\text {peak }}=0.436 \mathrm{mv}$ Vpeak $=0.421 \mathrm{mv}$ $V_{\text {peak }}=3.933 \mathrm{mv}$ $v_{\text {peak }}=0.389 \mathrm{mv}$ $V_{\text {peak }}=0.214 \mathrm{mv}$ $V_{\text {peak }}=0.275 \mathrm{mv}$ $V_{\text {peak }}=0.204 \mathrm{mv}$ $V_{\text {peak }}=0.186 \mathrm{mv}$ Vpeak $=6.306 \mathrm{mv}$
Prms $=0.661 \mathrm{E}-05 \mathrm{mw}$ Prms $=0.162 \mathrm{E}-04 \mathrm{mw}$ Prms $=0.145 E-04 \mathrm{mw}$ Prms $=0.269 E-05 \mathrm{mw}$ Prms $=0.251 \mathrm{E}-05 \mathrm{mw}$ Prms $=0.219 E-03 \mathrm{mw}$ Prms $=0.214 \mathrm{E}-0.5 \mathrm{mw}$ Prms $=0.646 \mathrm{E}-06 \mathrm{mw}$ Prms $=0.107 \mathrm{E}-05 \mathrm{mw}$ Prms $=0.589 \mathrm{E}-06 \mathrm{mw}$ Prms $=0.490 \mathrm{E}-06 \mathrm{mw}$ Prms $=0.562 E-03 \mathrm{mw}$
$\mathrm{dBm}=-51.8$ $\mathrm{dBm}=-47.9$ $\mathrm{dB} m=-48.4$ $\mathrm{dBm}=-55.7$ $\mathrm{dBm}=-56.0$ $\mathrm{dBm}=-36.6$ $\mathrm{dB} m=-56.7$ $\mathrm{dB} m=-61.9$ $\mathrm{dBm}=-59.7$ $\mathrm{dB} m=-62.3$ $\mathrm{dBm}=-63.1$ $\mathrm{dBm}=-32.5$

Table 2: $\frac{\Delta B}{B}$ calculated from frequerey spectrom measorements 


$\begin{array}{llllll}480 . & 0.0000001 & 200 . & 199.63 & 400.88 & 0.02 \\ 540 . & 0.0000002 & 200 . & 199.63 & 400.88 & 0.02 \\ 600 . & 0.0000001 & 200 . & 199.63 & 400.88 & 0.02 \\ 660 . & 0.0000000 & 200 . & 199.63 & 400.88 & 0.02 \\ 720 . & 0.0000015 & 200 . & 199.63 & 400.88 & 0.02\end{array}$

$\mathrm{B}=0.77$ tes la Frev $=616000$. hz GAMMAT $=5.54$

SIGMA BEAM $=140$. hz

$\begin{array}{rrllll}60 . & 0.0000030 & 140 . & 139.74 & 280.79 & 0.05 \\ 120 . & 0.0000023 & 140 . & 139.74 & 280.61 & 0.02 \\ 180 . & 0.0000015 & 140 . & 139.74 & 280.61 & 0.02 \\ 240 . & 0.0000005 & 140 . & 139.74 & 280.61 & 0.02 \\ 300 . & 0.0000004 & 140 . & 139.74 & 280.61 & 0.02 \\ 360 . & 0.0000029 & 140 . & 139.74 & 280.79 & 0.05 \\ 420 . & 0.0000002 & 140 . & 139.74 & 280.61 & 0.02 \\ 480 . & 0.0000001 & 140 . & 139.74 & 280.61 & 0.02 \\ 540 . & 0.0000001 & 140 . & 139.74 & 280.61 & 0.02 \\ 600 . & 0.0000001 & 140 . & 139.74 & 280.61 & 0.02 \\ 660 . & 0.0000001 & 140 . & 139.74 & 280.61 & 0.02 \\ 720 . & 0.0000023 & 140 . & 139.74 & 280.61 & 0.02\end{array}$

SIGMA BEAM $=160 . \mathrm{hz}$

$\begin{array}{rrllll}60 . & 0.0000030 & 160 . & 159.70 & 320.90 & 0.05 \\ 120 . & 0.0000023 & 160 . & 159.70 & 320.70 & 0.02 \\ 180 . & 0.0000015 & 160 . & 159.70 & 320.70 & 0.02 \\ -240 . & 0.0000005 & 160 . & 159.70 & 320.70 & 0.02 \\ 300 . & 0.0000004 & 160 . & 159.70 & 320.70 & 0.02 \\ 360 . & 0.0000029 & 160 . & 159.70 & 320.90 & 0.05 \\ 420 . & 0.0000002 & 160 . & 159.70 & 320.70 & 0.02 \\ 480 . & 0.0000001 & 160 . & 159.70 & 320.70 & 0.02 \\ 540 . & 0.0000001 & 160 . & 159.70 & 320.70 & 0.02 \\ 600 . & 0.0000001 & 160 . & 159.70 & 320.70 & 0.02 \\ 660 . & 0.0000001 & 160 . & 159.70 & 320.70 & 0.02 \\ 720 . & 0.0000023 & 160 . & 159.70 & 320.70 & 0.02\end{array}$

SIGMA BEAM $=180 . \mathrm{hz}$

$\begin{array}{rrllll}60 . & 0.0000030 & 180 . & 179.66 & 360.79 & 0.02 \\ 120 . & 0.0000023 & 180 . & 179.66 & 360.79 & 0.02 \\ 180 . & 0.0000015 & 180 . & 179.66 & 360.79 & 0.02 \\ 240 . & 0.0000005 & 180 . & 179.66 & 360.79 & 0.02 \\ 300 . & 0.0000004 & 180 . & 179.66 & 360.79 & 0.02 \\ 360 . & 0.0000029 & 180 . & 179.66 & 360.79 & 0.02 \\ 420 . & 0.0000002 & 180 . & 179.66 & 360.79 & 0.02 \\ 480 . & 0.0000001 & 180 . & 179.66 & 360.79 & 0.02 \\ 540 . & 0.0000001 & 180 . & 179.66 & 360.79 & 0.02 \\ 600 . & 0.0000001 & 180 . & 179.66 & 360.79 & 0.02 \\ 660 . & 0.0000001 & 180 . & 179.66 & 360.79 & 0.02 \\ 720 . & 0.0000023 & 180 . & 179.66 & 360.79 & 0.02\end{array}$

SIGMA BEAM $=200 . \mathrm{hz}$

$\begin{array}{rrllll}60 . & 0.0000030 & 200 . & 199.63 & 400.88 & 0.02 \\ 120 . & 0.0000023 & 200 . & 199.63 & 400.88 & 0.02 \\ 180 . & 0.0000015 & 200 . & 199.63 & 400.88 & 0.02 \\ 240 . & 0.0000005 & 200 . & 199.63 & 400.88 & 0.02 \\ 300 . & 0.0000004 & 200 . & 199.63 & 400.88 & 0.02 \\ 360 . & 0.0000029 & 200 . & 199.63 & 400.88 & 0.02\end{array}$




$\begin{array}{llllll} & & & & \\ & . & & & & \\ 420 . & 0.0000002 & 200 . & 199.63 & 400.88 & 0.02 \\ 480 . & 0.0000001 & 200 . & 199.63 & 400.88 & 0.02 \\ 540 . & 0.0000001 & 200 . & 199.63 & 400.88 & 0.02 \\ 600 . & 0.0000001 & 200 . & 199.63 & 400.88 & 0.02 \\ 660 . & 0.0000001 & 200 . & 199.63 & 400.88 & 0.02 \\ 720 . & 0.0000023 & 200 . & 199.63 & 400.88 & 0.02\end{array}$

